\title{
Topical Nanoemmigel Formulation of Boswellia serrata
}

\author{
H. KATHPALIA * AND K. K. SHREYA
}

Department of Pharmaceutics, Vivekanand Education Society's College of Pharmacy, Chembur, Mumbai-400 074 , India

Kathpalia and Shreya: Boswellia serrata Nanoemmigel

\begin{abstract}
Boswellia serrata was formulated as topical analgesic and antiinflammatory nanoemmigel, which is a combination of a nanoemulsion and a nanomicellar system in a gel base. Nanoemulsion was formulated by microemulsion-template method by using probe sonication at 5 amp for $5 \mathrm{~min}$. Particle size of nanoemulsion was found to be $141 \mathrm{~nm}$ and polydispersity index of 0.333 . Nanomicelle was formulated at a ratio 1:1 of drug and surfactant and 1:10 of solvent and antisolvent using solvent evaporation method. The particle size and polydispersity index of the nanomicelle was found to be $31.07 \mathrm{~nm}$ and 0.205 , respectively. Nanoemmigel was prepared by combining nanoemulsion and nanomicelle in the ratio 1:1 with Carbopol 974 as a gelling agent. The nanoemmigel prepared was a buff-coloured, opaque gel with smooth texture and good spreadability. Viscosity and drug content of the nanoemmigel was $50720 \mathrm{cps}$ and $103 \pm 1.08 \%$, respectively. In vitro diffusion study of nanoemmigel showed $98.52 \pm 1.53 \%$ drug permeation at the end of $5 \mathrm{~h}$. The drug release mechanism of the nanoemmigel formulation can be explained using the Higuchi model. The steady state flux and permeability co-efficient of the nanoemmigel was found to be better than each of nanoemulsion gel and nanomicellar gel due to the utilization of both the pathways available to the drug for permeation through the skin. Improved therapeutic response was obtained as compared to nanoemulsion gel, nanomicellar gel, emulgel and also marketed topical product of Boswellia when examined for in vivo antiinflammatory and analgesic activity in animal models
\end{abstract}

Key words: Boswellia serrata, nanoemulsion, nanomicelle, nanoemmigel, antiinflammatory, topical

Arthritis is a health problem with 1 in 5 people on an average, which affects their regular physical activities. For the treatment of arthritis and to reduce inflammation, a higher concentration of drug is required at the site of inflammation, which could be achieved by topical application directly on the target tissue ${ }^{[1]}$. The literature of Ayurveda describes various plants, to be used as whole, in parts or as extract for treating painful and inflammatory conditions like arthritis. Some of the herbs reported for use in arthritis were Capsicum annuum, Butea monosperma, Piper nigum, Gossypium herbaceum, Curcuma longa, Zingiber officinale, Camellia sinensis, Commiphora mukul, Boswellia serrata and Withania somnifera ${ }^{[2]}$.

B. serrata (N.O. Burseraceae) is a large, much branched, deciduous tree that grows abundantly in the dry, hilly parts of India. It is known as Dhup, Indian Frankincense or Indian Olibanum ${ }^{[3]}$. In India, the gum resin exudates of $B$. serrata, known in the vernacular as Salai guggal, has been used in the Ayurvedic system of medicine ${ }^{[4]}$ in the management of several inflammatory conditions. The major use of $B$. serrata

*Address for correspondence

in contemporary medicine is as an antiarthritic and antiinflammatory pharmacological agent.

B. serrata has poor oral bioavailability with an elimination half-life of $4.5 \pm 0.55 \mathrm{~h}$. Thus topical delivery of $B$. serrata is the preferred alternative to oral dosage form. But topical delivery is difficult due to its high lipophilicity $(\log \mathrm{P} 8)^{[5]}$. Many studies have demonstrated that molecules with intermediate lipophilicity $(\log \mathrm{P}, \mathrm{o} / \mathrm{w}$ of $2-3)$ can permeate via both the lipid and polar micro environments within the intercellular route ${ }^{[6]}$. To overcome the drawback of high lipophilicity, B. serrata has been formulated as nanoemulsion ${ }^{[7]}$, nanomicelle ${ }^{[1]}$ and nanoge ${ }^{[8]}$ so as to enhance permeability on topical application.

No study has been reported for it in nanoemmigel formulation, which utilizes the multiple absorption

This is an open access article distributed under the terms of the Creative Commons Attribution-NonCommercial-ShareAlike 3.0 License, which allows others to remix, tweak, and build upon the work non-commercially, as long as the author is credited and the new creations are licensed under the identical terms

Accepted 18 January 2018

Revised 11 May 2017

Received 29 December 2016

Indian J Pharm Sci 2018;80(2):261-267 
mechanism and has broad applicability. The hypothesis of the present work is that every nano drug delivery system is unique and its rate, extent and mechanism of action depend on the size, charge and composition of the nano drug delivery system. So, when a combination of completely different drug delivery systems is utilized for the delivery of drug, the absorption of the combined system would be better than either of the individual drug delivery systems due to utilization of the maximum possible absorption pathways ${ }^{[9]}$.

The present study was aimed to investigate $B$. serrata in novel topical nanoemmigel formulation consisting of a combination of nanoemulsion and nanomicelles to accomplish significant analgesic and antiinflammatory effect. This formulation was compared for in vitro diffusion and in vivo antiinflammatory and analgesic activity with nanoemulsion gel, nanomicellar gel, emulgel and marketed formulation (Sallaki ointment containing $7.5 \% \mathrm{w} / \mathrm{w}$ B. serrata, manufactured by Gufic Biosciences).

\section{MATERIALS AND METHODS}

B. serrata extract was obtained from Synthite Industry Ltd., Mumbai. Cremophor EL, polysorbate 80, polysorbate 20, Kolliphor 188, Kolliphor 407, Solutol HS 15 and Kolliphor TPGS were obtained as gift samples from BASF India Ltd., Mumbai, India. Laurogylcol 90, Capryol 90, Capryol PGMC, Capmul, Labrafac PG and Labrafac lipophill were obtained as gift samples from Gattefosse, Mumbai. Carbopol 974 was obtained as gift sample from Lubrizol advanced materials Inc., Mumbai. All other ingredients and solvents were purchased from Loba Chemie, Mumbai.

\section{Solubility evaluation:}

The solubility of $B$. Serrata extract in various oils, $10 \% \mathrm{w} / \mathrm{v}$ surfactant solutions and in co-surfactants was determined by shake flask method. Briefly, an excess amount of extract was mixed with aforementioned liquids and then shaken using orbital flask shaker at $37^{\circ}$ for $48 \mathrm{~h}$. Samples were centrifuged at $5000 \mathrm{rpm}$ for $10 \mathrm{~min}$ and subsequently filtered through $0.22 \mu \mathrm{m}$ syringe driven membrane filter unit. The filtrates were assayed by UV-spectrophotometer (Shimadzu, 1800 double beam spectrophotometer) at $249 \mathrm{~nm}$ to evaluate the amount of drug dissolved ${ }^{[10]}$.

\section{Screening of surfactants and co-surfactants:}

Initial evaluation of emulsifying properties was carried out by visual assessment. Various surfactant and co-surfactants in 2:1 ratio were mixed. Then oil was added to the above mixture in the ratio of $1: 3(\mathrm{w} / \mathrm{w})$, heated and vortexed gently to form homogeneous mixtures. About $500 \mathrm{mg}$ of this mixture was dispersed into $10 \mathrm{ml}$ of water with gentle stirring and then assessed visually for self-emulsification in terms of dispersibility and ease of emulsification ${ }^{[10,11]}$.

\section{Pseudo-ternary phase diagram:}

On the basis of solubility studies and emulsification studies, isopropyl myristate (IPM, oil), polysorbate 80 (surfactant), isopropyl alcohol (IPA, co-surfactant) and distilled water were selected to prepare microemulsion. For determining the microemulsion region pseudoternary phase diagrams were constructed for different combinations of oil and $\mathrm{S}_{\text {mix }}(2: 8,3: 7,4: 6,5: 5,6: 4,7: 3$, $8: 2$ ) at weight ratios of $1: 1,2: 1,3: 1$ of surfactant and co-surfactant $\left(\mathrm{S}_{\text {mix }}\right)$.

\section{Formulation of nanoemulsion using microemulsion template method:}

$B$. serrata microemulsion was prepared by dissolving extract into oily phase separately. Surfactant and co-surfactant in the 3:1 ratio were mixed with oily phase to form a pre-concentrate, which was sonicated for about 10 min to get a clear solution. Required amount of water was added under stirring followed by probe sonication at $5 \mathrm{amp}$ up to $5 \mathrm{~min}$ to get nanoemulsion. The nanoemulsion was stored at room temperature until used.

\section{Formulation of nanomicelles:}

B. serrata nanomicelles were prepared with Cremophor EL using solvent evaporation method. The Cremophor EL and $B$. serrata in the ratio $1: 1$ were dissolved in methanol to which distilled water was added (1:10) under continuous stirring to form nanomicelles followed by evaporation of solvent.

\section{Globule size analysis of the nanoemulsion and} nanomicelles:

The average globule size and polydispersity index (PI) of the nanoemulsion and nanomicelles were determined using a Zetasizer 1000 HS (Malvern Instruments, Worcestershire, UK). Samples were diluted with double-distilled water to ensure that the light-scattering intensity was within the instrument's sensitivity range. Double-distilled water was filtered through $0.45 \mu \mathrm{m}$ membrane filters prior to globule size determination. 
Formulation of $B$. serrata nanoemulsion gel, nanomicelle gel and nanoemmigel:

Each of nanoemulsion and nanomicelle was formulated into gel using $1.5 \%$ Carbopol 974 as a gelling agent (Table 1). About $1.5 \%$ of Carbopol 974 was soaked in distilled water and then stirred continuously at low rpm to get a uniform gel free of lumps and air bubbles. Nanoemulsion and nanomicelles formulation (1:1) were incorporated into the carbopol gel with uniform mixing. The $\mathrm{pH}$ was then neutralized with triethanolamine to get nanoemmigel (Table 1).

\section{Formulation of $\boldsymbol{B}$. serrata emulgel:}

About $1 \%$ of Carbopol 974 was soaked in distilled water and then stirred continuously at low rpm to get a uniform gel free of lumps and air bubbles. B. serrata extract was dissolved in IPM and polysorbate 80, which was then incorporated into the carbopol gel with uniform mixing. The $\mathrm{pH}$ was then neutralized with triethanolamine to get emulgel (Table 1).

Determination of drug content, spreadability, pH and viscosity of nanoemmigel:

For determining drug content, about $1 \mathrm{~g}$ of gel was dissolved in $100 \mathrm{ml}$ of methanol. It was filtered through Whatman filter paper and analysed using a UV spectrophotometer at $249 \mathrm{~nm}$. Spreadability of the gel was determined by pressing $0.5 \mathrm{~g}$ of the gel between two tiles. Initial diameter of gel was noted. A constant weight of $2 \mathrm{~kg}$ was kept on the tiles assembly for $2 \mathrm{~min}$ and the increase in diameter was noted ${ }^{[12]}$. The $\mathrm{pH}$ of the $1 \% \mathrm{w} / \mathrm{w}$ gel was determined using DBK digital $\mathrm{pH}$ meter, calibrated using $\mathrm{pH} 4.0$ and 7.0 standard buffers before use ${ }^{[8]}$. Viscosity of the gel was determined using Brookfield viscometer employing T-shaped spindle (S94) at $2 \mathrm{rpm}$.
In vitro diffusion study using egg membrane and release kinetics:

The in vitro diffusion study was carried out through egg membrane using Franz diffusion cell ${ }^{[13]}$. The diffusion medium consisted of phosphate buffer $\mathrm{pH} 6.8$ and IPA in the ratio of 40:60. The formulation equivalent to $25 \mathrm{mg}$ of drug was applied on $3.46 \mathrm{~cm}^{2}$ area of egg membrane mounted at the lower end of donor compartment. The donor and receptor compartments were held together using a clamp. The receptor compartment containing $13 \mathrm{ml}$ of diffusion medium was maintained at $37 \pm 0.5^{\circ}$ and stirred with magnetic stirrer to prevent the formation of concentrated drug solution layer below the diffusion membrane. Samples of $2 \mathrm{ml}$ were collected at predetermined time intervals $(1,2,3,4$ and $5 \mathrm{~h}$ ) and replaced with fresh buffer. The concentration of drug was determined using a UV spectrophotometer at $241 \mathrm{~nm}$. The cumulative amount of drug permeated across the egg membrane was calculated and plotted against time ${ }^{[14,15]}$. Data obtained from in vitro release studies were fitted to various kinetics equations (zero order, first order, Higuchi and Korsmeyer-Peppas models) to find out the mechanism of drug release from nanoemmigel.

In vivo antiinflammatory study using carrageenaninduced rat paw oedema:

The guidelines of the Institutional Animal Ethics Committee (approval no. VESCOP/03/2015c) were followed for the pharmacodynamic studies. Female Wistar rats weighing 160-200 g were allowed to adapt the environment $\left(24 \pm 1^{\circ}\right.$ and 12:12 h dark:light cycles). Food and water were made available ad libitum. Before the experiment, the animals were fasted overnight. The formulation was applied topically on dorsal part of hind paw. After $60 \mathrm{~min}, 0.1 \%$ carrageenan in

TABLE 1: COMPOSITION OF VARIOUS FORMULATIONS

\begin{tabular}{|c|c|c|c|c|}
\hline \multirow{2}{*}{ Formulations/ingredients } & Nanoemulsion gel & Nanomicelle gel & Nanoemmigel & Emulgel \\
\hline & \multicolumn{4}{|c|}{ Amount added (\% w/w) } \\
\hline Boswellia serrata extract & 5 & 5 & 5 & 5 \\
\hline Isopropyl myristate & 7 & 7 & 7 & 7 \\
\hline Polysorbate 80 & 33.75 & - & 33.75 & 33.75 \\
\hline Isopropyl alcohol & 11.25 & - & 11.25 & - \\
\hline Cremophor EL & - & 5 & 5 & - \\
\hline Carbopol 974 & 1.5 & 1.5 & 1.5 & 1 \\
\hline Triethanolamine & q.s to $\mathrm{pH} 7$ & q.s to $\mathrm{pH} 7$ & q.s to $\mathrm{pH} 7$ & q.s to $\mathrm{pH} 7$ \\
\hline Methyl paraben & 0.18 & 0.18 & 0.18 & 0.18 \\
\hline Propyl paraben & 0.02 & 0.0 & 0.02 & 0.02 \\
\hline Distilled water & q.s to 100 & q.s to 100 & q.s to 100 & q.s to 100 \\
\hline
\end{tabular}

q.s: Quantity sufficient

March-April 2018 
isotonic solution was injected into the plantar region of the left hind paw. The contra lateral paw injected was used as a control. The volume difference between the carrageenan and control paws was used to evaluate the inflammatory response. Paw volume was measured using water plethysmometer immediately and 1, 2, 3, 4 and $5 \mathrm{~h}$ after carrageenan injection ${ }^{[16]}$.

\section{In vivo analgesic study using Eddy's hot plate:}

The analgesic activity of the formulations was evaluated using the hot plate method that employed an electronically controlled hot plate heated to $55^{\circ}( \pm 0.1)$. Each rat was placed unrestrained on the hot plate for baseline measurement just prior to drug application and considered as zero time or control. About $100 \mathrm{mg}$ of gel was applied topically to the hind paw of the rat and the gel remaining on the surface of the skin was wiped off with a piece of cotton after $30 \mathrm{~min}$. Measurements of pain threshold for the treated animals were taken after $0.5,1,1.5,2,2.5$ and $3 \mathrm{~h}$ after drug application. Latency to lift and/or licking a hind paw or attempt to jump from the apparatus was recorded for the control and drug-treated groups ${ }^{[17]}$. The cut-off time was $30 \mathrm{~s}$ to avoid tissue damage from exposure to hot plate.

\section{Skin irritation study:}

The skin irritation study was carried out on female Wistar rats using the Draize patch test. Six animals were used in each group. A single dose of formulation was applied on the left side of the rat, with the right side as control. The development of erythema was monitored after $24 \mathrm{~h}$ and $14 \mathrm{~d}^{[18-20]}$.

\section{Stability studies:}

Stability of the nanoemmigel was evaluated under accelerated stability conditions $\left(40 \pm 2^{\circ} / 75 \pm 5 \% \mathrm{RH}\right)$ in a stability testing chamber for 3 mo. Physical characteristics and the drug content of the samples were evaluated initially and at the end of 3 mo of storage period.

\section{RESULTS AND DISCUSSION}

Solubility studies were mainly carried out for selection of suitable excipients for preparing nanoemulsion and nanomicelles of $B$. serrata. Identifying suitable oil showing maximum solubilizing potential for drug and surfactant/co-surfactant showing good emulsification property is very important to achieve optimum drug loading. Solubility studies help mainly in selection of oily phase. It was found from literature that, unmodified edible oils are the logical and preferred lipid excipients; however, they exhibit relatively poor emulsification efficiency. Criteria for selection, was that oil should show ease of emulsification as well as highest solubility of drug. Therefore, solubility studies of the extract were carried out in semi-synthetic, modified oils (fig. 1). Isopropyl myristate, divya dhara oil and Labrafac PG showed comparatively more solubility of extract than the rest of the oils. Isopropyl myristate showing maximum solubility of $B$. serrata extract was selected as the oily phase for microemulsion.

The solubility of $B$. serrata extract in various surfactants and co-surfactants is shown in figs. 2A and $\mathrm{B}$, respectively. B. serrata exhibited solubility in the order of cremophor EL>polysorbate $20>$ Solutol HS $>$ Kolliphor TPGS $>$ Kolliphor P 407>Polysorbate $80>$ Kolliphor 188 . The non-ionic surfactants used insolubility studies are known to be less irritant and cytotoxic than anionic and cationic surfactants. Cremophor EL showing high solubility for B. serrata was selected for the preparation of nanomicelle. Among the co-surfactants, IPA showed the highest solubility of $B$. serrata. Selection of surfactants and co-surfactants was primarily governed by their emulsification efficiency for the selected oily phase. From the screening study of surfactants and co-surfactants by assessment of dispersion properties, it was found that the combination of polysorbate 80 and IPA exhibited excellent emulsification ability for IPM and hence it was selected for the preparation of microemulsion (Table 2).

The pseudo ternary phase diagram of IPM-polysorbate 80: IPA-water microemulsion system is shown in fig. 3 , in which white circle in the black coloured region of microemulsion indicates microemulsion composition selected for formulation based on the solubility of $5 \%$. serrata extract. The amount of surfactant and co-surfactant $\left(\mathrm{S}_{\text {mix }}\right)$ was selected on the basis of

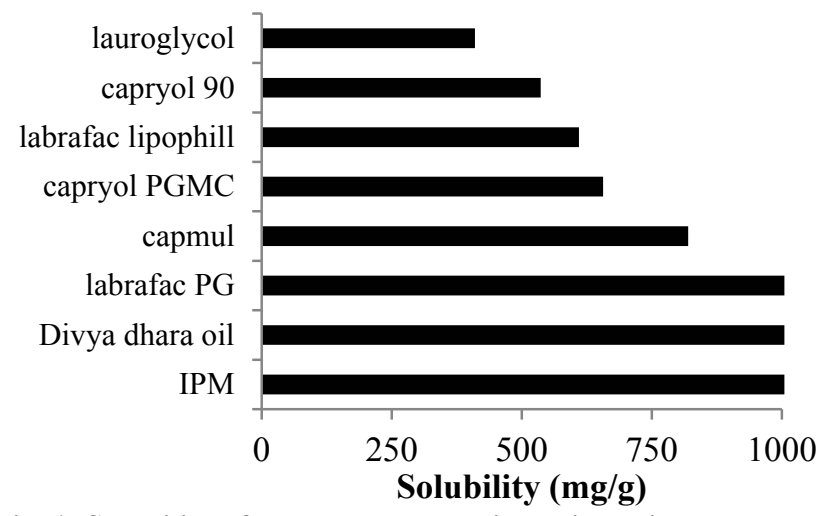

Fig. 1: Solubility of $B$. serrata extract in various oily phases 
minimum amount of $\mathrm{S}_{\text {mix }}$ required for emulsification and maximum amount of water incorporation possible. The globule size of the nanoemulsion formed by probe sonication of microemulsion and nanomicelles were found to be 141.8 and $31.07 \mathrm{~nm}$, respectively and PI of 0.333 and 0.205 , respectively. PI indicates that the formulations prepared were fairly monodispersed as it was below 0.5 and near to zero.

The boswellic acid content of nanoemmigel, nanoemulsion gel, nanomicelle gel, emulgel and

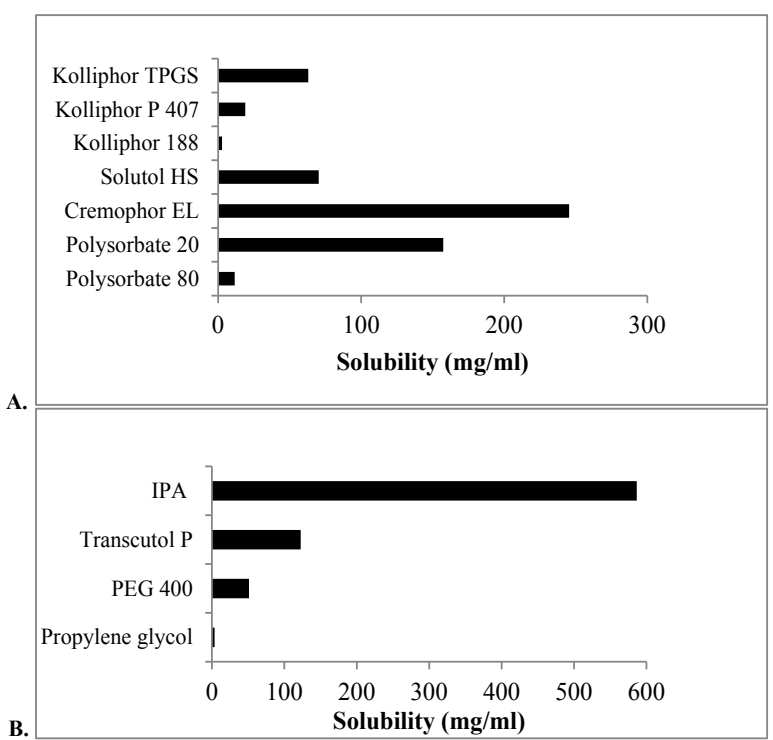

Fig. 2: Solubility of $B$. serrata extract in various surfactants (A) and co-surfactants (B)

TABLE 2: EMULSIFICATION EFFICIENCIES OF SURFACTANT-CO-SURFACTANT COMBINATIONS $(2: 1 \mathrm{w} / \mathrm{w})$

\begin{tabular}{lccc}
\hline Oil & Surfactant & Co-surfactant & Observation \\
\hline IPM & Cremophor EL & Propylene Glycol & + \\
IPM & Cremophor EL & PEG 400 & + \\
IPM & Cremophor EL & Transcutol P & + \\
IPM & Cremophor EL & Polysorbate 80 & - \\
IPM & IPA & Polysorbate 80 & - \\
IPM & Polysorbate 80 & Transcutol P & + \\
IPM & Polysorbate 80 & Cremophor EL & - \\
IPM & Polysorbate 80 & IPA & ++ \\
\hline
\end{tabular}

++ Very good, compositions spread rapidly in water forming clear and transparent emulsion; + good, compositions droplets spread in water to form turbid emulsion; - poor, compositions do not spread in water and form coalescence of oil droplets marketed Sallaki ointment was found to be within limits. Spreadability is an important property of topical formulation for patient compliance. High spreadability values of nanoemmigel indicate better spreading ability at the site of application as compared to other formulations studied. $\mathrm{pH}$ of these formulations was found in the range 6.5 to 7.1 , which is compatible with the skin. Viscosity values were found to be in the range of 47 000-69 $000 \mathrm{cps}$ for all the formulation. Nanoemmigel exhibited pseudo-plastic flow with thixotropic property (Table 3 ).

In vitro diffusion studies were performed to ensure drug release. The release profiles of boswellic acid from different formulations are illustrated in fig. 4. Nanoemmigel showed maximum diffusion due to the maximum possible paths available for the transport of drug. Nanoemmigel showed significant higher flux and permeability co-efficient than other formulations (Table 4). The difference between the release pattern of nanoemmigel and marketed formulation was significant as determined by $f 2$ test $(f 2=23)$. The drugrelease mechanism was studied by comparing the respective correlation coefficients for different release models. It was observed that the drug release was

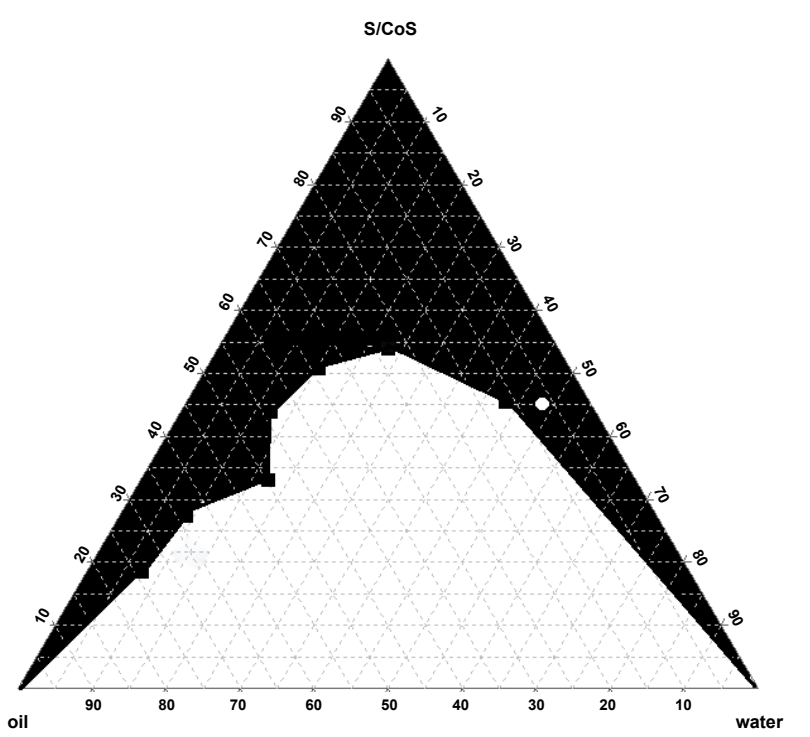

Fig. 3: Pseudo ternary phase diagram of microemulsion system White circle in the dark region indicates the selected composition

TABLE 3: DRUG CONTENT, pH, SPREADABILITY AND VISCOSITY OF VARIOUS FORMULATIONS

\begin{tabular}{lcccc}
\hline \multirow{2}{*}{ Formulation } & \multicolumn{3}{c}{ Parameters } \\
\cline { 2 - 5 } & Drug content (\%) & pH & Spreadability (cm) & Viscosity (cps) \\
\hline Nanoemulsion gel & $99.99 \pm 1.25$ & 6.5 & $5.15 \pm 0.20$ & 47100 \\
Nanomicelle gel & $99.55 \pm 1.43$ & 6.8 & $4.30 \pm 0.20$ & 58100 \\
Nanoemmigel & $103.03 \pm 1.08$ & 6.9 & $5.50 \pm 0.13$ & 50720 \\
Emulgel & $102.45 \pm 0.41$ & 6.7 & $4.75 \pm 0.18$ & 67800 \\
Marketed formulation & $98.25 \pm 1.34$ & 7.1 & $4.07 \pm 0.12$ & 68600 \\
\hline
\end{tabular}




\begin{tabular}{lccccc}
\hline \multirow{2}{*}{ Parameter } & \multicolumn{5}{c}{ Formulations } \\
\cline { 2 - 6 } & Nanoemulsion gel & Nanomicelle gel & Nanoemmigel & Emulgel & Marketed formulation \\
\hline Cumulative release (\%) & $91.47 \pm 1.75$ & $87.18 \pm 1.58$ & $98.52 \pm 1.53$ & $70.67 \pm 3.46$ & $81.09 \pm 1.98$ \\
after $5 \mathrm{~h}$ & 1.32 & 1.25 & 1.43 & 1.02 & 1.17 \\
Flux $\left(\mathrm{mg} / \mathrm{cm}^{2} / \mathrm{h}\right.$ ) & 0.0528 & 0.0500 & 0.0572 & 0.0408 & 0.0468 \\
Permeation coefficient & 52 & 70 & 23 & 52 & - \\
$(\mathrm{cm} / \mathrm{h})$ & 15 & 5 & 58 & 15 & - \\
Similarity factor (f2) & & & &
\end{tabular}

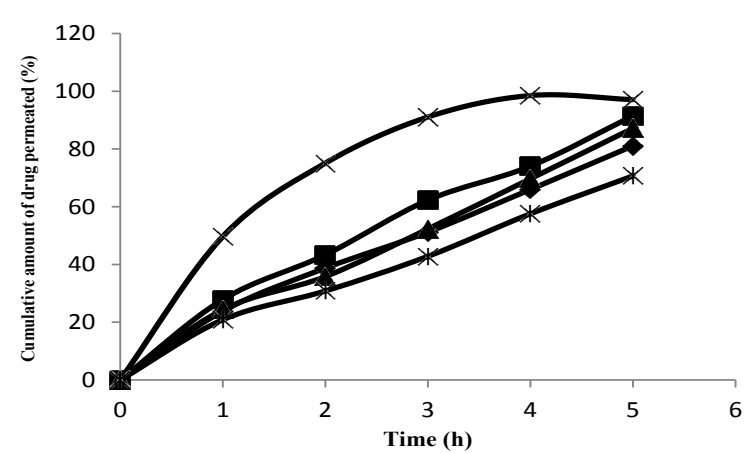

Fig. 4: In vitro diffusion profile $(\mathrm{n}=3)$

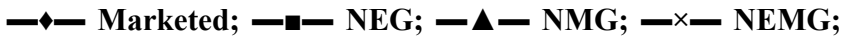
$\longrightarrow \mid<-$ emulgel

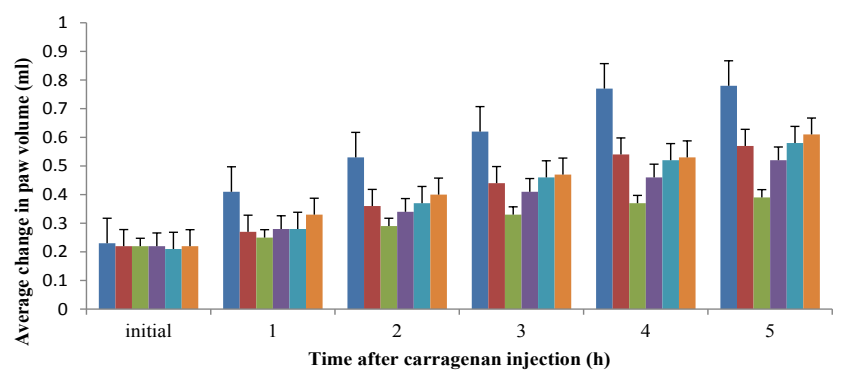

Fig. 5: Antiinflammatory effect of various formulations

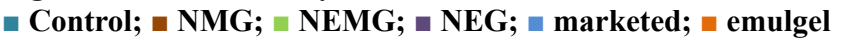

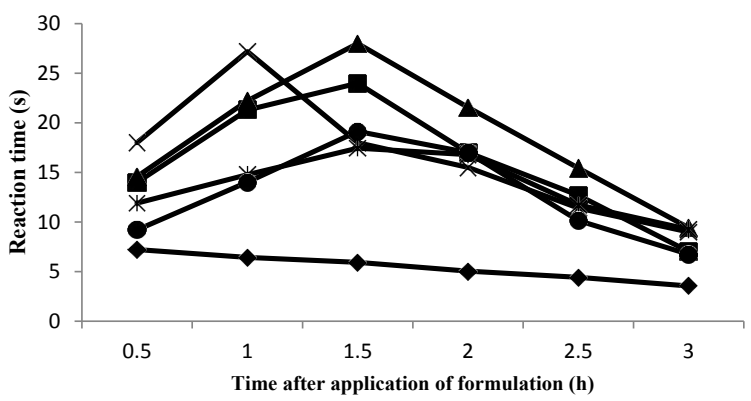

Fig. 6: Analgesic effect of various formulations

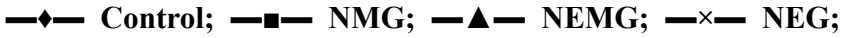
$\rightarrow \mid<-$ marketed; -०- emulgel

diffusion-controlled in the nanoemmigel formulation $\left(\mathrm{R}^{2}=0.971\right)$.

The intra-planter injection of carrageenan into the hind paw in rats induced an increase in the paw volume. This oedema had a rapid onset and reached a peak at $5 \mathrm{~h}$ after the challenge. Pre-treatment with nanoemmigel containing boswellic acid and marketed ointment resulted in 50 and $25.64 \%$ inhibition in paw oedema volume, respectively at $5 \mathrm{~h}$ as compared to control group $(\mathrm{p}<0.05)$ and fig. 5 showed the average change in volume at different time intervals for $5 \mathrm{~h}$. Nanoemmigel containing boswellic acids produced significant antiinflammatory effect as compared to nanoemulsion gel, nanomicelle gel, emulgel and marketed formulation.

In vivo analgesic study using Eddy's hot plate demonstrated that nanoemmigel showed higher analgesic activity as compared to control group and marketed ointment group $(\mathrm{p}<0.05)$ most probably due to enhanced permeation of boswellic acids through the skin (fig. 6).

All the formulations and marketed ointment did not show any sign of erythema or oedema during the skin irritation study even after $24 \mathrm{~h}$ and $14 \mathrm{~d}$ indicating their safety for topical application.

Nanoemmigel formulation was stable for at least three months at accelerated condition $\left(40 \pm 2^{\circ} / 75 \pm 5 \% \mathrm{RH}\right)$ as no significant change in appearance, consistency and drug content was observed.

Herbal nanoemmigel formulation of $B$. serrata showed improved analgesic and antiinflammatory activities that could be attributed to better skin permeation due to utilization of the both paracellular and transcellular pathways available for absorption of the active ingredients. Nanoemmigel appears to have the potential to be considered as a promising novel drug delivery system for enhancing drug permeation through skin for local action into the inflamed tissues.

\section{Acknowledgements:}

The authors would like to thank the BASF, Lubrizol and Gattefosse, India for providing the excipients. They also thank the Principal, VES College of Pharmacy, Mumbai for providing the required facilities to carry out this research work. 


\section{Conflicts of interest:}

There are no conflicts of interest.

\section{Financial support and sponsorship:}

Nil.

\section{REFERENCES}

1. Goel A, Ahmad FJ, Singh RM, Singh GN. 3-acetyl-11-ketobeta-boswellic acid loaded-polymeric nanomicelles for topical anti-inflammatory and anti-arthritic activity. J Pharm Pharmacol 2010;62:273-78.

2. Ranade SY, Gaud RS. Current strategies in herbal drug delivery for arthritis: An overview. Int J Pharm Sci Res 2013;4:3782-94.

3. Sharma A, Bhatia S, Kharya MD, Gajbhiye V, Ganesh N, Namdeo AG, et al. Anti-inflammatory and analgesic activity of different fraction of Boswellia serrate. Int $\mathrm{J}$ Phytomed 2010;2:94-9.

4. The Ayurvedic Pharmacopoeia of India. Part I, Vol. IV. New Delhi: Department of AYUSH, Government of India Ministry of Health and Family Welfare; 2008. p. 57-8.

5. Krüger P, Daneshfar R, Eckert GP, Klein J, Volmer DA, Bahr U, et al. Metabolism of boswellic acids in vitro and in vivo. Drug Metab Dispos 2008;6:1135-42.

6. Benson HAE. Skin structure, function and permeation In. Benson HAE, Watkinson AC, editors. Topical and Transdermal Drug Delivery: Principles and Practice. New Jersey: John Wiley and Sons, Inc.; 2011. p. 3-22.

7. Gohel M, Soni T, Hingorani L, Patel A, Patel N. Development and optimization of plant extract loaded nanoemulsion mixtures for the treatment of inflammatory disorder. Curr Res Drug Discov 2014;1:29-38.

8. Goel A, Ahmad FJ, Singh RM, Singh GN. Anti-inflammatory activity of nanogel formulation of 3-acetyl-11-keto- $\beta$ boswellic acid. Pharmacologyonline 2009;3:311-18.

9. Somagoni J, Boakye CHA, Godugu C, Patel AR, Faria HAM,
Zucolotto V, et al. Nanoemmigel- A novel drug delivery system for topical application- In vitro and in vivo evaluation. PlosONE 2014;9:1-17.

10. Borhade V, Pathak S, Sharma S, Patravale V. Clotrimazole nanoemulsion for malaria chemotherapy. Part I: preformulation studies, formulation design and physicochemical evaluation. Int J Pharm 2012;431:138-48.

11. Pouton CW, Formulation of Poorly Water-Soluble Drugs for Oral Administration: Physicochemical and Physiological Issues and the Lipid Formulation Classification System. Eur J Pharm Sci 2006;2:278-87.

12. Waghmare N, Waghmare P, Wani S, Yerawar A. Gel for the treatment of acne vulgaris. Res J Pharm 2011;2:220.

13. Nawale R, Mayee R. Behaviour of natural membrane on drug permeation. Int J Pharm Innov 2013;3:45-54.

14. Kumar DS, Sairam R, Anandbabu S, Karpagavalli L, Maheswaran A, Narayanan N. Formulation and evaluation of transdermal patches of salbutamol. Res J Pharm Biol Chem Sci 2012;3:1482-94.

15. Singla S, Harikumar SL, Aggrawal G. Proniosomes for effective topical delivery of clotrimazole: development, characterization and performance evaluation. Asian J Pharm Sci 2012;7:259-70.

16. Bachhav YG, Patravale VB. Formulation of meloxicam gel for topical application: in vitro and in vivo evaluation. Acta Pharm 2010;60:153-63.

17. Fathy AI, Hamdy DM, Mansour A, Sami AM. Evaluation of the antiinflammatory and analgesic effects of piroxicam loaded microemulsion in topical formulations. Int $\mathrm{J}$ Pharm Pharm Sci 2011;3:66-70.

18. Ammar HO, Ghorab M, El-Nahhas SA, Kamel R. Polymeric matrix system for prolonged delivery of tramadol hydrochloride, part ii: biological evaluation. AAPS Pharm Sci Tech 2009;10:1065.

19. https://ntp.niehs.nih.gov/iccvam/suppdocs/feddocs/oecd/ oecdtg404.pdf.

20. Prajapati BG, Patel MM. Cross-linked chitosan gel for local drug delivery of clotrimazole. J Sci Technol 2010;5:43-52. 